\title{
Quadratic fields with infinite Hilbert 2-class field towers
}

\author{
by \\ Frank GerTh III (Austin, TX)
}

1. Introduction. Let $K$ be a finite extension field of the rational number field $\mathbb{Q}$, and let $C_{K}$ be the 2 -class group of $K$ (i.e., the Sylow 2 -subgroup of the ideal class group of $K$ ) in the usual sense. Let $K_{1}$ be the Hilbert 2-class field of $K$ (i.e., the maximal abelian unramified extension of $K$ whose Galois group is a 2-group), and let $K_{i}$ be the Hilbert 2-class field of $K_{i-1}$ for $i \geq 2$. Then

$$
K \subset K_{1} \subset \ldots \subset K_{i} \subset \ldots
$$

is the Hilbert 2-class field tower of $K$. If $K_{i} \neq K_{i-1}$ for all $i$, then the Hilbert 2 -class field tower is said to be infinite.

Next we define the 2-class rank and the 4-class rank of $K$. Let $C_{K}^{i}=$ $\left\{a^{i}: a \in C_{K}\right\}$. We define the 2-class rank $r_{K}$ by

$$
r_{K}=\operatorname{rank} C_{K}=\operatorname{dim}_{\mathbb{F}_{2}}\left(C_{K} / C_{K}^{2}\right)
$$

where $\mathbb{F}_{2}$ is the finite field with two elements, and we are viewing the elementary abelian 2-group $C_{K} / C_{K}^{2}$ as a vector space over $\mathbb{F}_{2}$. We define the 4-class rank $s_{K}$ by

$$
s_{K}=\operatorname{rank} C_{K}^{2}=\operatorname{dim}_{\mathbb{F}_{2}}\left(C_{K}^{2} / C_{K}^{4}\right) .
$$

We note that $0 \leq s_{K} \leq r_{K}$.

Now suppose $K$ is an imaginary quadratic extension of $\mathbb{Q}$. It is known (cf. [1, p. 233]) that the Hilbert 2-class field tower of $K$ is infinite if $r_{K} \geq 5$. We shall prove some results for the cases where $r_{K}=3$ or 4 . For nonnegative integers $r$ and $s$, square-free positive integers $m$, and positive real numbers $x$, we define

$$
\begin{aligned}
V_{r} & =\left\{K=\mathbb{Q}(\sqrt{-m}): \text { the } 2 \text {-class rank } r_{K}=r\right\}, \\
V_{r ; x} & =\left\{K=\mathbb{Q}(\sqrt{-m}) \in V_{r}: m \leq x\right\}, \\
V_{r, s ; x} & =\left\{K \in V_{r ; x}: \text { the } 4 \text {-class rank } s_{K}=s\right\}, \\
V_{r, s ; x}^{*} & =\left\{K \in V_{r, s ; x}: \text { the Hilbert 2-class field tower of } K \text { is infinite }\right\} .
\end{aligned}
$$


Then we define a density

$$
\delta_{r, s}^{*}=\liminf _{x \rightarrow \infty} \frac{\left|V_{r, s ; x}^{*}\right|}{\left|V_{r ; x}\right|}
$$

where $|V|$ denotes the cardinality of a finite set $V$. We shall prove the following theorem in Section 2 of this paper.

Theorem 1. For imaginary quadratic fields let $\delta_{r, s}^{*}$ be defined by (3). Then $\delta_{3, s}^{*}>0$ for $1 \leq s \leq 3$ and $\delta_{4, s}^{*}>0$ for $0 \leq s \leq 4$.

REMARK. Thus a positive proportion of the imaginary quadratic fields with 2-class rank equal to 3 have infinite Hilbert 2-class field towers and 4-class rank equal to $s$ for each value $s=1,2$, and 3. Similarly, a positive proportion of the imaginary quadratic fields with 2-class rank equal to 4 have infinite Hilbert 2-class field towers and 4-class rank equal to $s$ for each value $s=0,1,2,3$, and 4 .

REMARK. An essential part of the proof of Theorem 1 depends on Corollary 3 in a paper of Hajir [6]. In fact, Theorem 1 can be viewed as a generalization of ideas introduced in [6].

Now suppose $K$ is a real quadratic extension of $\mathbb{Q}$. From $[1$, p. 233] the Hilbert 2-class field tower of $K$ is infinite if $r_{K} \geq 6$. We shall prove some results for the cases where $r_{K}=4$ or 5 . For nonnegative integers $r$ and $s$, square-free integers $m>1$, and positive real numbers $x$, we define

$$
\begin{aligned}
W_{r} & =\left\{K=\mathbb{Q}(\sqrt{m}): \text { the 2-class rank } r_{K}=r\right\}, \\
W_{r ; x} & =\left\{K=\mathbb{Q}(\sqrt{m}) \in W_{r}: m \leq x\right\}, \\
W_{r, s ; x} & =\left\{K \in W_{r ; x}: \text { the } 4 \text {-class rank } s_{K}=s\right\}, \\
W_{r, s ; x}^{*} & =\left\{K \in W_{r, s ; x}: \text { the Hilbert 2-class field tower of } K \text { is infinite }\right\},
\end{aligned}
$$

and

$$
\varepsilon_{r, s}^{*}=\liminf _{x \rightarrow \infty} \frac{\left|W_{r, s ; x}^{*}\right|}{\left|W_{r ; x}\right|} .
$$

We shall prove the following theorem in Section 3 of this paper.

Theorem 2. For real quadratic fields let $\varepsilon_{r, s}^{*}$ be defined by (4). Then $\varepsilon_{4, s}^{*}>0$ for $0 \leq s \leq 4$ and $\varepsilon_{5, s}^{*}>0$ for $0 \leq s \leq 5$.

REMARK. So a positive proportion of the real quadratic fields with 2class rank equal to 4 have infinite Hilbert 2-class field towers and 4-class rank equal to $s$ for each value $s=0,1,2,3$, and 4 . Similarly, a positive proportion of the real quadratic fields with 2-class rank equal to 5 have infinite Hilbert 2-class field towers and 4-class rank equal to $s$ for each value $s=0,1,2,3,4$, and 5 . 
2. Proof of Theorem 1. Let $K$ be an imaginary quadratic field in which exactly $t$ finite primes are ramified, where $t$ is a positive integer. From genus theory we know that the 2-class rank $r_{K}$ equals $t-1$. With $t=r+1$, we see that the set $V_{r ; x}$ in this paper is the same as the set $A_{t ; x}$ in [4]. So from equation (2.5) in [4],

$$
\left|V_{r ; x}\right| \sim \frac{1}{2} \cdot \frac{1}{r !} \cdot \frac{x(\log \log x)^{r}}{\log x} \quad(\text { as } x \rightarrow \infty) .
$$

Now suppose $K=\mathbb{Q}\left(\sqrt{-p_{1} \ldots p_{t}}\right)$, where $p_{1}<\ldots<p_{t}$ are primes with $p_{i} \equiv 1(\bmod 4)$ for $1 \leq i \leq t-1$ and $p_{t} \equiv 3(\bmod 4)$. From equations $(2.6)$ and (2.7) in [4], the 4-class rank $s_{K}$ satisfies

$$
s_{K}=t-1-\operatorname{rank} M_{K}
$$

where $M_{K}$ is a $t \times t$ matrix over $\mathbb{F}_{2}$ whose entries $a_{i j}$ are defined by Legendre symbols as follows:

$$
(-1)^{a_{i j}}= \begin{cases}\left(\frac{P_{j}}{p_{i}}\right) & \text { if } i \neq j, \\ \left(\frac{\bar{P}_{j}}{p_{i}}\right) & \text { if } i=j,\end{cases}
$$

with $P_{j}=p_{j}$ if $p_{j} \equiv 1(\bmod 4), P_{j}=-p_{j}$ if $p_{j} \equiv 3(\bmod 4)$, and $\bar{P}_{j}$ $=-p_{1} \ldots p_{t} / P_{j}$. From quadratic reciprocity and properties of Legendre symbols, the matrix $M_{K}$ is completely determined by the set of values $\left\{\left(\frac{p_{j}}{p_{i}}\right)\right.$ for $\left.1 \leq i<j \leq t\right\}$. For positive real numbers $x$, let

$$
\begin{aligned}
S(K, t ; x)=\{ & \mathbb{Q}\left(\sqrt{-p_{1}^{\prime} \ldots p_{t}^{\prime}}\right) \text { with primes } p_{1}^{\prime}<\ldots<p_{t}^{\prime}, \\
& p_{i}^{\prime} \equiv p_{i}(\bmod 4) \text { for } 1 \leq i \leq t, \\
& \left.\left(\frac{p_{j}^{\prime}}{p_{i}^{\prime}}\right)=\left(\frac{p_{j}}{p_{i}}\right) \text { for } 1 \leq i<j \leq t, \text { and } p_{1}^{\prime} \ldots p_{t}^{\prime} \leq x\right\} .
\end{aligned}
$$

From equation (2.12) in [4],

$$
|S(K, t ; x)| \sim 2^{-\left(t^{2}+t\right) / 2} \cdot \frac{1}{(t-1) !} \cdot \frac{x(\log \log x)^{t-1}}{\log x} \quad(\text { as } x \rightarrow \infty) .
$$

The proof of this formula depends on character sum estimates similar to those used in Section 4 of [3] and Section 5 of [5]. Alternatively, one can use the analytic machinery developed in [2]. Note that from (5) and with $t=r+1$ in (8), we get

$$
\lim _{x \rightarrow \infty} \frac{|S(K, r+1 ; x)|}{\left|V_{r ; x}\right|}=2^{-\left(r^{2}+3 r\right) / 2}>0 .
$$


The fact that this limit is positive will be a key part of the proof of Theorem 1. However, first we need the following lemma, which follows from Corollary 3 in $[6]$.

Lemma 1. Suppose $K=\mathbb{Q}\left(\sqrt{-p_{1} \ldots p_{t}}\right)$ with $p_{1}, \ldots, p_{t}$ distinct primes and $t \geq 4$. Also suppose $p_{1} \equiv p_{2} \equiv 1(\bmod 4)$ and $\left(\frac{p_{j}}{p_{i}}\right)=1$ for $i=1,2$ and $j=3,4$. Then the Hilbert 2-class field tower of $K$ is infinite.

Proof. Let $F=\mathbb{Q}\left(\sqrt{p_{1}}, \sqrt{p_{2}}\right)$. Since $p_{1} \equiv p_{2} \equiv 1(\bmod 4)$ and $\left(\frac{p_{j}}{p_{i}}\right)=1$ for $i=1,2$ and $j=3,4, p_{3}$ and $p_{4}$ split completely in the totally real degree 4 extension $F$ of $\mathbb{Q}$. Then by Corollary 3 in [6], $E=F K$ has infinite Hilbert 2-class field tower. Since $E$ is contained in the Hilbert 2-class field of $K$, the Hilbert 2-class field tower of $K$ is infinite.

Now consider $K=\mathbb{Q}\left(\sqrt{-p_{1} p_{2} p_{3} p_{4}}\right)$ with primes $p_{1}<p_{2}<p_{3}<p_{4}$ such that $p_{i} \equiv 1(\bmod 4)$ for $1 \leq i \leq 3$ and $p_{4} \equiv 3(\bmod 4)$. Then the 2-class rank $r_{K}$ equals 3 .

CASE $s=1$ : Suppose $\left(\frac{p_{j}}{p_{i}}\right)=1$ for $i=1,2$ and $j=3,4 ;\left(\frac{p_{2}}{p_{1}}\right)=-1$; $\left(\frac{p_{4}}{p_{3}}\right)=-1$. From Lemma $1, K$ has an infinite Hilbert 2-class field tower, and from (7) one can check that $\operatorname{rank} M_{K}=2$. Then the 4-class rank $s_{K}$ is 1 from (6). Furthermore every field in the set $S(K, 4 ; x)$ has an infinite Hilbert 2-class field tower and 4-class rank equal to 1 . So $S(K, 4 ; x) \subset V_{3,1 ; x}^{*}$, and then equations (3) and (9) imply $\delta_{3,1}^{*}>0$.

CASE $s=2$ : Suppose $\left(\frac{p_{j}}{p_{i}}\right)=1$ for $i=1,2$ and $j=3,4 ;\left(\frac{p_{2}}{p_{1}}\right)=-1$; $\left(\frac{p_{4}}{p_{3}}\right)=1$. Then a similar analysis shows $S(K, 4 ; x) \subset V_{3,2 ; x}^{*}$, and then $\delta_{3,2}^{*}>0$.

Case $s=3$ : Suppose $\left(\frac{p_{j}}{p_{i}}\right)=1$ for $1 \leq i<j \leq 4$. Then $S(K, 4 ; x) \subset$ $V_{3,3 ; x}^{*}$, and $\delta_{3,3}^{*}>0$.

Now consider $K=\mathbb{Q}\left(\sqrt{-p_{1} p_{2} p_{3} p_{4} p_{5}}\right)$ with primes $p_{1}<p_{2}<p_{3}<p_{4}<p_{5}$ such that $p_{i} \equiv 1(\bmod 4)$ for $1 \leq i \leq 4$ and $p_{5} \equiv 3(\bmod 4)$. Then the 2-class rank $r_{K}$ equals 4 .

CASE $s=0$ : Suppose $\left(\frac{p_{j}}{p_{i}}\right)=1$ for $1 \leq i<j \leq 4$ and $\left(\frac{p_{5}}{p_{i}}\right)=-1$ for $1 \leq i \leq 4$. Then $S(K, 5 ; x) \subset V_{4,0 ; x}^{*}$, and $\delta_{4,0}^{*}>0$.

CASES $1 \leq s \leq 3$ : If $s=1,2$, or 3 , suppose $\left(\frac{p_{j}}{p_{i}}\right)=1$ for $1 \leq i<j \leq 4$; $\left(\frac{p_{5}}{p_{i}}\right)=1$ for $1 \leq i \leq s ;\left(\frac{p_{5}}{p_{i}}\right)=-1$ for $s+1 \leq i \leq 4$. Then $S(K, 5 ; x) \subset V_{4, s ; x}^{*}$ and $\delta_{4, s}^{*}>0$.

CASE $s=4$ : Suppose $\left(\frac{p_{j}}{p_{i}}\right)=1$ for $1 \leq i<j \leq 5$. Then $S(K, 5 ; x) \subset$ $V_{4,4 ; x}^{*}$, and $\delta_{4,4}^{*}>0$.

Thus the proof of Theorem 1 is complete. 
REMARK. It is known that $V_{3,3 ; x}^{*}=V_{3,3 ; x}$ and $V_{4, s ; x}^{*}=V_{4, s ; x}$ for $s=3,4$ (see [6] and [7]). One could use these facts to give an alternative proof that $\delta_{3,3}^{*}>0, \delta_{4,3}^{*}>0$, and $\delta_{4,4}^{*}>0$.

3. Proof of Theorem 2. Let $K=\mathbb{Q}(\sqrt{m})$, where $m>1$ is a squarefree integer. Let $r_{K}$ be the 2-class rank of $K$, and let $t$ be the number of primes that ramify in $K / \mathbb{Q}$. It is well known that

$$
r_{K}= \begin{cases}t-1 & \text { if no prime dividing } m \\ t-2 & \text { is congruent to } 3(\bmod 4), \\ & \text { is at least one prime dividing } m\end{cases}
$$

For nonnegative integers $r$ and positive real numbers $x$, we let

$$
\begin{aligned}
Y_{r ; x}=\{ & k=\mathbb{Q}(\sqrt{m}): m=p_{1} \ldots p_{r+2} \leq x \\
& \text { with odd primes } p_{1}<\ldots<p_{r+2} \\
& \text { and with a positive even number of } \left.p_{i} \equiv 3(\bmod 4)\right\} .
\end{aligned}
$$

If $N_{x}$ is the number of square-free positive integers $m \leq x$ with $r+2$ prime factors, then

$$
N_{x} \sim \frac{1}{(r+1) !} \cdot \frac{x(\log \log x)^{r+1}}{\log x} \quad(\text { as } x \rightarrow \infty)
$$

(see [8, Theorem 437]). If $N_{\mathrm{e}, x}$ is the number of square-free positive integers $m \leq x$ with $r+2$ prime factors and an even number of these primes congruent to $3(\bmod 4)$, then $N_{\mathrm{e}, x} \sim \frac{1}{2} N_{x}$. In $Y_{r ; x}$, we are excluding the set

$$
\left\{m=p_{1} \ldots p_{r+2} \leq x \text { with each } p_{i} \equiv 1(\bmod 4)\right\},
$$

which has cardinality asymptotic to $2^{-(r+2)} N_{x}$. We are also excluding the set

$$
\left\{m=p_{1} \ldots p_{r+2} \leq x \text { with } 2 \mid m\right\},
$$

which has cardinality $o\left(N_{x}\right)$. So

$$
\left|Y_{r ; x}\right| \sim\left(\frac{1}{2}-\frac{1}{2^{r+2}}\right) \cdot \frac{1}{(r+1) !} \cdot \frac{x(\log \log x)^{r+1}}{\log x} \quad(\text { as } x \rightarrow \infty) .
$$

Now recall that $W_{r ; x}=\left\{K=\mathbb{Q}(\sqrt{m})\right.$ : 2-class rank $r_{K}$ equals $r$ and $m \leq x\}$. We note that

$$
\left|W_{r ; x}\right| \sim\left|Y_{r ; x}\right| \quad(\text { as } x \rightarrow \infty)
$$

since $Y_{r ; x} \subset W_{r ; x}$ and the set of elements of $W_{r ; x}$ that are not in $Y_{r ; x}$ has cardinality $o\left(\left|Y_{r ; x}\right|\right)$. Then for nonnegative integers $s$, we define

$$
Y_{r, s ; x}^{*}=\left\{K \in Y_{r ; x}: \text { the 4-class rank } s_{K}=s\right. \text {, and the }
$$

Hilbert 2-class field tower of $K$ is infinite\}. 
Then from (4) and the above discussion, we get

$$
\varepsilon_{r, s}^{*}=\liminf _{x \rightarrow \infty} \frac{\left|Y_{r, s ; x}^{*}\right|}{\left|Y_{r ; x}\right|} .
$$

Now suppose $K=\mathbb{Q}\left(\sqrt{p_{1} \ldots p_{t}}\right)$, where $p_{1}<\ldots<p_{t}$ are primes with $p_{i} \equiv 1(\bmod 4)$ for $1 \leq i \leq t-2$ and $p_{t-1} \equiv p_{t} \equiv 3(\bmod 4)$. Then the 2 -class rank $r_{K}$ is $t-2$. For the 4-class rank, we shall use results from Section 5 of [4]. However, first we remark that the 2-class groups considered in Section 5 of [4] are the narrow 2-class groups. For the field $K$ that we are considering, the narrow 2-class rank is $t-1$ rather than $t-2$, but the narrow 4-class rank and the usual 4-class rank are the same. Hence from equations (5.5) and (5.6) in [4], the 4-class rank $s_{K}$ satisfies

$$
s_{K}=t-1-\operatorname{rank} M_{K}
$$

where $M_{K}$ is the $t \times t$ matrix over $\mathbb{F}_{2}$ whose entries $a_{i j}$ satisfy

$$
(-1)^{a_{i j}}= \begin{cases}\left(\frac{P_{j}}{p_{i}}\right) & \text { if } i \neq j, \\ \left(\frac{\bar{P}_{j}}{p_{i}}\right) & \text { if } i=j,\end{cases}
$$

with $P_{j}=p_{j}$ if $p_{j} \equiv 1(\bmod 4), P_{j}=-p_{j}$ if $p_{j} \equiv 3(\bmod 4)$, and $\bar{P}_{j}=$ $p_{1} \ldots p_{t} / P_{j}$. Note that $\operatorname{rank} M_{K} \geq 1$ since either $\left(\frac{P_{t-1}}{p_{t}}\right)=-1$ or $\left(\frac{P_{t}}{p_{t-1}}\right)=$ -1 by quadratic reciprocity since $p_{t-1} \equiv p_{t} \equiv 3(\bmod 4)$. So $s_{K} \leq t-2$. Furthermore, from quadratic reciprocity and properties of Legendre symbols, the matrix $M_{K}$ is completely determined by the set of values $\left\{\left(\frac{p_{j}}{p_{i}}\right)\right.$ for $1 \leq i<j \leq t\}$. For positive real numbers $x$, let

$$
\begin{aligned}
S^{\prime}(K, t ; x)= & \left\{\mathbb{Q}\left(\sqrt{p_{1}^{\prime} \ldots p_{t}^{\prime}}\right) \text { with primes } p_{1}^{\prime}<\ldots<p_{t}^{\prime},\right. \\
& p_{i}^{\prime} \equiv p_{i}(\bmod 4) \text { for } 1 \leq i \leq t, \\
& \left.\left(\frac{p_{j}^{\prime}}{p_{i}^{\prime}}\right)=\left(\frac{p_{j}}{p_{i}}\right) \text { for } 1 \leq i<j \leq t, \text { and } p_{1}^{\prime} \ldots p_{t}^{\prime} \leq x\right\} .
\end{aligned}
$$

Then analogously to (8) we have

$$
\left|S^{\prime}(K, t ; x)\right| \sim 2^{-\left(t^{2}+t\right) / 2} \cdot \frac{1}{(t-1) !} \cdot \frac{x(\log \log x)^{t-1}}{\log x} \quad(\text { as } x \rightarrow \infty) .
$$

From (12) and (16) with $t=r+2$, we get

$$
\lim _{x \rightarrow \infty} \frac{\left|S^{\prime}(K, r+2 ; x)\right|}{\left|Y_{r ; x}\right|}=2^{-(r+1)(r+2) / 2} \cdot\left(2^{r+1}-1\right)^{-1}>0 .
$$

Now we prove a lemma analogous to Lemma 1. 
Lemma 2. Suppose $K=\mathbb{Q}\left(\sqrt{p_{1} \ldots p_{t}}\right)$ with distinct primes $p_{1}, \ldots, p_{t}$ and $t \geq 6$. Also suppose $p_{1} \equiv p_{2} \equiv 1(\bmod 4)$ and $\left(\frac{p_{j}}{p_{i}}\right)=1$ for $i=1,2$ and $j=3,4,5$. Then the Hilbert 2-class field tower of $K$ is infinite.

Proof. Since $\left(\frac{p_{j}}{p_{i}}\right)=1$ for $i=1,2$ and $j=3,4,5$, we see that $p_{3}, p_{4}$, and $p_{5}$ split completely in $F=\mathbb{Q}\left(\sqrt{p_{1}}, \sqrt{p_{2}}\right)$. Also $p_{6}$ splits in at least one of the subfields $\mathbb{Q}\left(\sqrt{p_{1}}\right), \mathbb{Q}\left(\sqrt{p_{2}}\right), \mathbb{Q}\left(\sqrt{p_{1} p_{2}}\right)$ of $F$. Let $L=F\left(\sqrt{p_{3} \ldots p_{t}}\right)$. Then there are at least 14 ramified primes in $L / F$. Let $E_{F}$ be the group of units in the ring of algebraic integers in $F$, and let $E_{F}^{2}=\left\{u^{2}: u \in E_{F}\right\}$. Since $\operatorname{dim}_{\mathbb{F}_{2}}\left(E_{F} / E_{F}^{2}\right)=4$, from genus theory the 2-class rank $r_{L}$ satisfies

$$
r_{L} \geq 14-1-4=9 \text {. }
$$

From [1, p. 233], $L$ has an infinite Hilbert 2-class field tower if $r_{L} \geq 2+$ $2 \sqrt{\gamma_{L}+1}$, where $\gamma_{L}$ is the number of infinite primes of $L$. Since $\gamma_{L}=8$, we get $r_{L} \geq 9>2+2 \sqrt{8+1}$, and thus $L$ does have an infinite Hilbert 2-class field tower. Since $L$ is contained in the Hilbert 2-class field of $K$, we conclude that $K$ has an infinite Hilbert 2-class field tower.

Now consider $K=\mathbb{Q}\left(\sqrt{p_{1} \ldots p_{6}}\right)$ with primes $p_{1}<\ldots<p_{6}$ such that $p_{i} \equiv 1(\bmod 4)$ for $1 \leq i \leq 4$ and $p_{5} \equiv p_{6} \equiv 3(\bmod 4)$. Then the $2-$ class $\operatorname{rank} r_{K}$ equals 4 . Analogously to the procedure we used in proving Theorem 1, we list conditions on Legendre symbols that imply that the 4-class rank $s_{K}$ equals $s$ for a given value of $s$ (by (14) and (15)) and so that $K$ has an infinite Hilbert 2-class field tower (by Lemma 2). Then we get $S^{\prime}(K, 6 ; x) \subset Y_{4, s ; x}^{*}$, and using (13) and (17), we get $\varepsilon_{4, s}^{*}>0$.

CASE $s=0$ : Suppose $\left(\frac{p_{j}}{p_{i}}\right)=1$ for $1 \leq i<j \leq 5$; $\left(\frac{p_{6}}{p_{i}}\right)=-1$ for $1 \leq i \leq 5$.

CASES $1 \leq s \leq 4$ : For $s=1,2,3$, or 4 , suppose $\left(\frac{p_{j}}{p_{i}}\right)=1$ for $1 \leq i<j$ $\leq 5 ;\left(\frac{p_{6}}{p_{i}}\right)=1$ for $1 \leq i \leq s ;\left(\frac{p_{6}}{p_{i}}\right)=-1$ for $s+1 \leq i \leq 5$.

Next consider $K=\mathbb{Q}\left(\sqrt{p_{1} \ldots p_{7}}\right)$ with primes $p_{1}<\ldots<p_{7}$ such that $p_{i} \equiv 1(\bmod 4)$ for $1 \leq i \leq 5$ and $p_{6} \equiv p_{7} \equiv 3(\bmod 4)$. Then the 2-class rank $r_{K}$ equals 5 . We will get $S^{\prime}(K, 7 ; x) \subset Y_{5, s ; x}^{*}$ and $\varepsilon_{5, s}^{*}>0$ if we choose the primes as follows:

CASE $s=0$ : Suppose $\left(\frac{p_{j}}{p_{i}}\right)=1$ for $1 \leq i<j \leq 6 ;\left(\frac{p_{7}}{p_{i}}\right)=-1$ for $1 \leq i \leq 6$.

CASEs $1 \leq s \leq 5$ : If $s=1,2,3,4$, or 5 , suppose $\left(\frac{p_{j}}{p_{i}}\right)=1$ for $1 \leq i<$ $j \leq 6 ;\left(\frac{p_{7}}{p_{i}}\right)=1$ for $1 \leq i \leq s ;\left(\frac{p_{7}}{p_{i}}\right)=-1$ for $s+1 \leq i \leq 6$.

Then the proof of Theorem 2 is complete.

REMARK. It is known that $W_{4,4 ; x}^{*}=W_{4,4 ; x}$ and $W_{5, s ; x}^{*}=W_{5, s ; x}$ for $s=4,5$ (see [9]). One could use these facts to give an alternative proof that $\varepsilon_{4,4}^{*}>0, \varepsilon_{5,4}^{*}>0$, and $\varepsilon_{5,5}^{*}>0$. 


\section{References}

[1] J. W. S. Cassels and A. Fröhlich, Algebraic Number Theory, Academic Press, London, 1986.

[2] J. Cremona and R. Odoni, Some density results for negative Pell equations: an application of graph theory, J. London Math. Soc. 39 (1989), 16-28.

[3] F. Gerth, Counting certain number fields with prescribed $\ell$-class numbers, J. Reine Angew. Math. 337 (1982), 195-207.

[4] —, The 4-class ranks of quadratic fields, Invent. Math. 77 (1984), 489-515.

[5] - Densities for ranks of certain parts of p-class groups, Proc. Amer. Math. Soc. 99 (1987), 1-8.

[6] F. Hajir, On a theorem of Koch, Pacific J. Math. 176 (1996), 15-18.

[7] -, Correction to "On a theorem of Koch", ibid. 196 (2000), 507-508.

[8] G. Hardy and E. Wright, An Introduction to the Theory of Numbers, 4th ed., Oxford Univ. Press, London, 1965.

[9] C. Maire, Un raffinement du théorème de Golod-Safarevic, Nagoya Math. J. 150 (1998), 1-11.

Department of Mathematics

The University of Texas at Austin

Austin, TX 78712-1082, U.S.A.

E-mail: gerth@math.utexas.edu

Received on 15.6.2001

and in revised form on 15.3.2002 